\title{
Automatic human signature recognition system
}

\author{
E. Da Sacco ${ }^{1}$, F. Garzia ${ }^{1,2}$ \& R. Cusani ${ }^{1}$ \\ ${ }^{1}$ Department of Information, Electronics and Telecommunication \\ Engineering - SAPIENZA - University of Rome, Italy \\ ${ }^{2}$ Wessex Institute of Technology, Southampton, UK
}

\begin{abstract}
In the last years biometry has shown a growing interest and it is used in a plenty of applications and devices. The term biometry derives from the Greek term "bios" (life) and "metros" (to measure) and it represents the science that studies the mathematical relations between human behaviors or human physical features.

A specific branch of biometrics is represented by human signature recognition that allows to associate each person to his sign.

The purpose of this paper is to present an automatic human signature system based on genetic algorithms (GAs).

Keywords: biometry, human signature recognition, genetic algorithms.
\end{abstract}

\section{Introduction}

Human signature recognition is an extremely interesting branch of biometry that allows to solve a plenty of security identification problem [1-12].

Human signature is reasonably unique not only from the esthetical point of view but also from the features (writing velocity, curves, etc.) point of view that belongs to the behavioral sphere and that are just inimitable.

If signature is not made on paper but on an electronic pad, it is possible to extract the behavioral features.

Signature recognition is very useful in economic and bank money transactions where signing is a frequent activity that does not require the final user to be trained and increase the security level.

Biometrics signature recognition uses a certain number of features both static and dynamic. Static features are related to the signature itself such as the height/width ratio, the position of the centroid of the sign, the crossing point, the curved lines, the terminal points and so on. Dynamic features are acquirable by 
means of proper devices such as electronic pads. They are represented by writing velocity, writing pressure, angle of inclination of the pen, acceleration, number of time that the pen is raised up and so on. Both the categories (static and dynamic) are very useful for signature recognition: if from one side one could think that dynamic features are better from the descriptive point of view, on the other side if the signature is already made on a document, it is possible to consider only static parameter. The considered features depend, therefore, on the particular application.

The great advantage of signature recognition is represented by its great acceptability from the user that does note any difference in normal signing and biometric signing, since these operations are very familiar.

One disadvantage is represented by the instability of human signature that can vary with time and this variation must be considered in the used biometric technique.

The proposed system uses only static features.

\section{The proposed system}

The proposed system operates on human signature, acquired by means of every kind of device, extracting for each signature seven features that represent the essence of the signature (template) and that are illustrated in the following.

The seven features (template) represent an array in a seven dimensional space. Since these arrays can be very similar for a set of signatures, it is necessary to find an optimal base in the seven dimensional space to increase the difference between vectors and to correctly classify the different arrays representing the signatures of the different persons stored in the database. The research of the optimal base in the seven dimensional space was made by means of Genetic Algorithms [13] that use the principle of natural evolution and natural selection to solve optimization problems that can be modeled by the mathematical point of view.

The proposed system operates according to the scheme shown in fig. 1.

From fig. 1 it is possible to see that the system extracts the signature features and store them in an array that is properly reduced thanks to a Principal Components Analysis (PCA) transformation. After PCA transformation, a proper whitening transformation is applied to optimize the signature parameters. After whitening transformation a proper rotation transformation using GAs is applied to increase the distance of the optimized array of signature features and to increase the discrimination power of the system to ensure a high recognition rate with minimal error.

\section{The pre-processing}

The preprocessing is necessary to prepare the signatures acquired from paper, for example by means of a scanner, to be processed by the system.

The original image is scanned from paper in gray scale (256 levels) and converted properly in a black/white scale. 


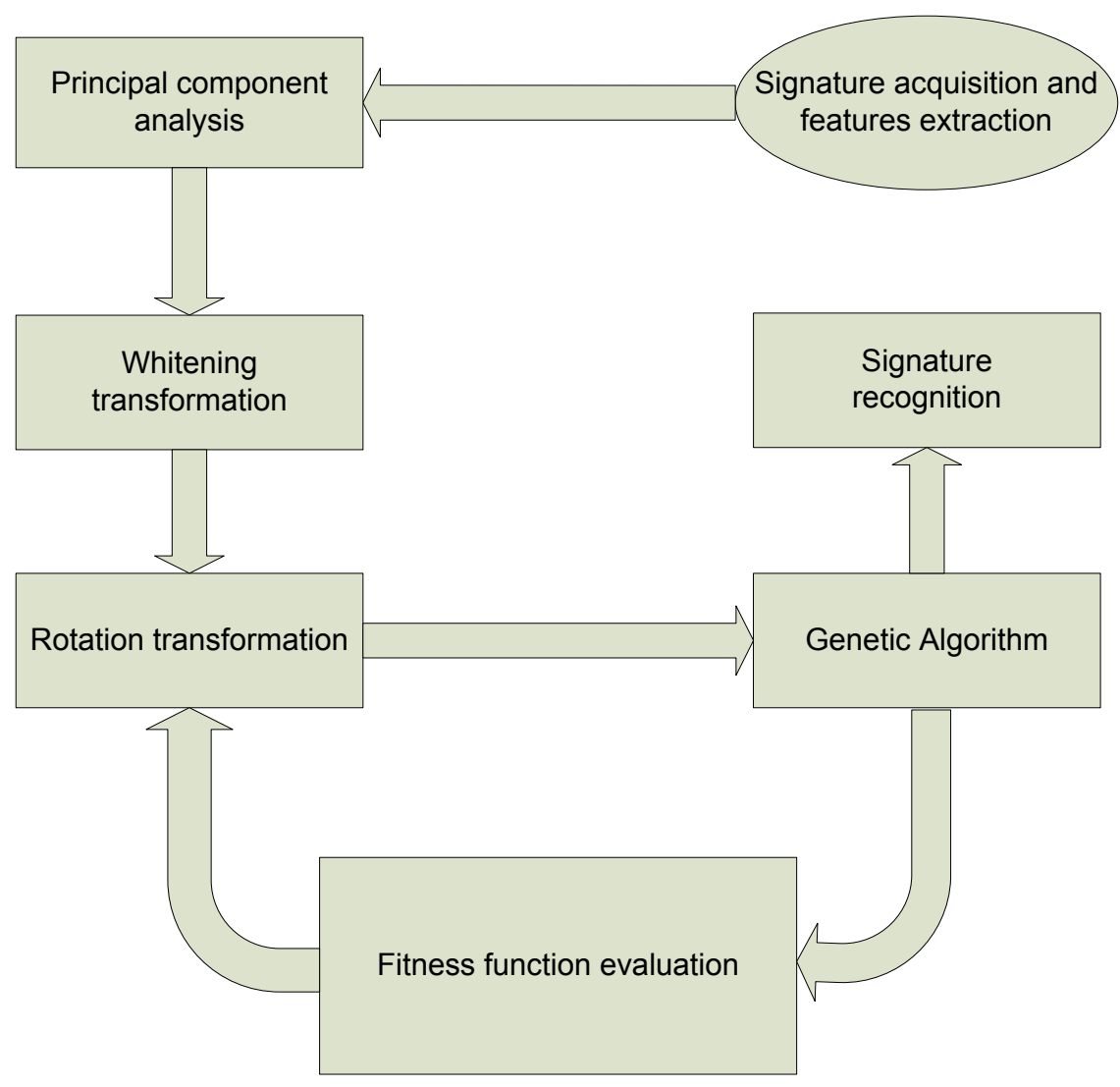

Figure 1: Operative scheme of the system.

The conversion algorithm is quite critical to obtain good results.

It could be possible to use a classical method where a proper threshold is used: pixels above the threshold are converted in white pixels while pixels below the threshold are converted in black pixels. This technique is not optimal for our purpose since results are quite rough, as shown in figs $2-3$.

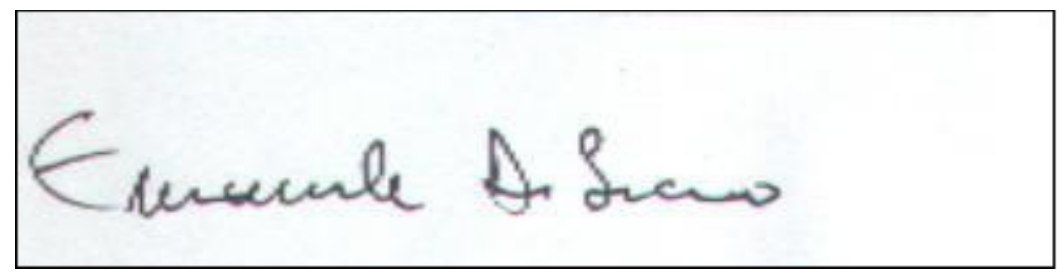

Figure 2: $\quad$ Original gray scale signature. 


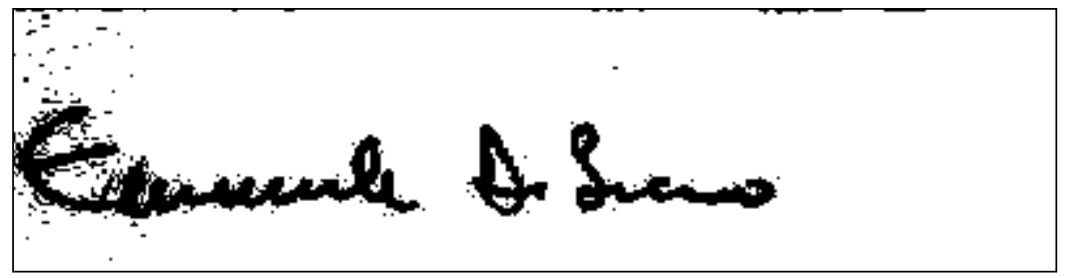

Figure 3: Black/white version of the signature using the classical method.

To obtain a good level of conversion it is necessary to use a more refined method such as Otsu [14] algorithm, whose results are shown in fig. 4.

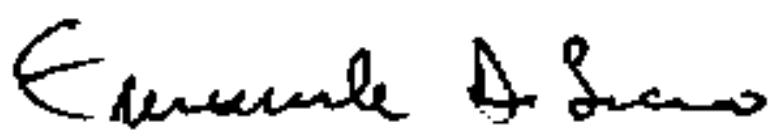

Figure 4: Black/white version of the signature using the Otsu algorithm.

\section{Signature features extraction}

Features are extremely important for signature recognition.

The proposed technique is very flexible and allows us to use the desired number of features. For this reason only fundamental features are used and the obtained results are already excellent with a reduce number of parameters.

The first operation to do is represented by the calculation of the 4 extremes points of the signature as shown in fig. 5 .

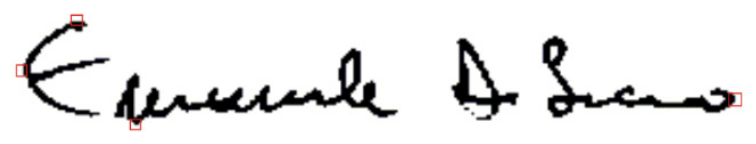

Figure 5: $\quad$ The 4 extremes point of the signature.

The basic feature to extract is represented by the centroid of the signature, calculated considering the envelope of the signature itself, as shown in fig. 6 . 


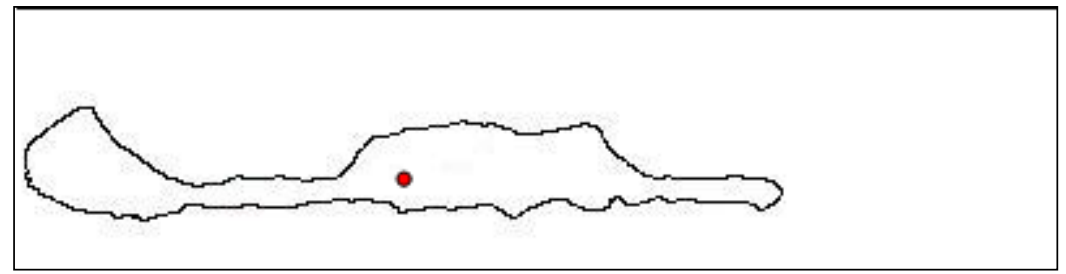

Figure 6: The centroid of the signature.

The first feature to extract is represented by the ratio $\mathrm{H}_{\mathrm{W}}$ between the height $\mathrm{H}$ and the width $\mathrm{W}$ :

as shown in fig. 7.

$$
\mathrm{H}_{\mathrm{W}}=\mathrm{H} / \mathrm{W}
$$

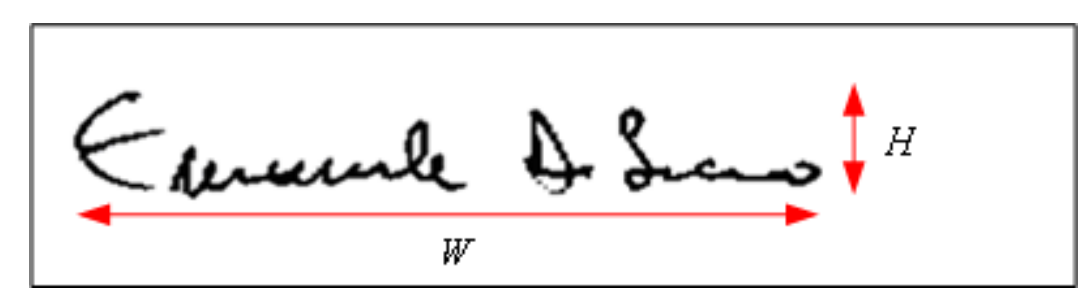

Figure 7: The ratio $\mathrm{H}_{\mathrm{W}}=\mathrm{H} / \mathrm{W}$.

The second feature to extract is represented by the ratio $\mathrm{L}_{\mathrm{W}}$ between the number pix of black pixels and the width W:

$$
L w=\frac{p i x}{W}
$$

as shown in fig. 8 .

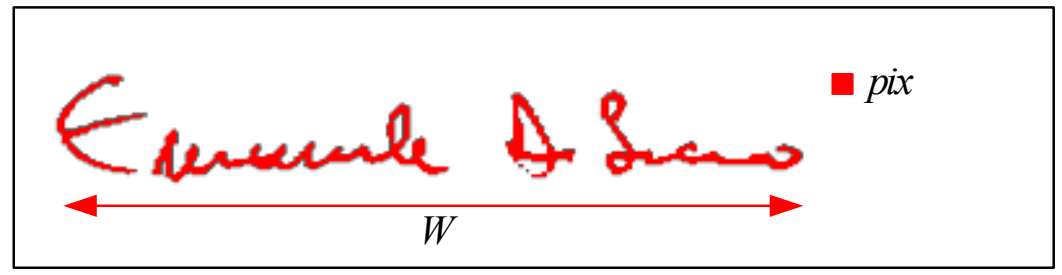

Figure 8: $\quad$ The ratio $\mathrm{L}_{\mathrm{W}}=$ pix/W.

The third feature to extract is represented by the distance $X_{\mathrm{cn}}$ between the mean horizontal point $X_{\mathrm{m}}$ and the left most point $X_{\mathrm{w}}$ of the signature:

$$
X c n=X_{m}-X_{w}
$$

as shown in fig. 9 . 


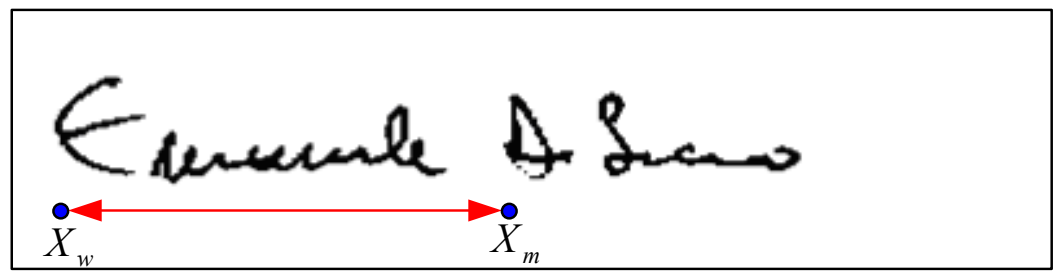

Figure 9: The distance $X_{c n}$ between the mean point of the signature $X_{m}$ and the left most point $X_{\mathrm{w}}$ of the signature.

The fourth parameter to extract is represented by the distance between vertical mean point $Y_{m}$ and the upper most point $Y_{w}$ of the signature:

$$
Y c n=Y_{m}-Y_{w}
$$

as shown in fig. 10 .

$$
\text { Emumale A Lano: }
$$

Figure 10: The distance $Y_{c n}$ between the mean point of the signature $Y_{m}$ and the upper most point $\mathrm{Y}_{\mathrm{w}}$ of the signature.

The fifth parameter to extract is represented by the ratio LR:

$$
L R=\frac{X_{c e n}-X_{w}}{X_{e}-X_{c e n}}
$$

where $X_{\text {cen }}$ is the transversal coordinate of the centroid, $X_{w}$ is the most left point of the signature, $X_{e}$ is the most right point of the signature, as shown in fig. 11.

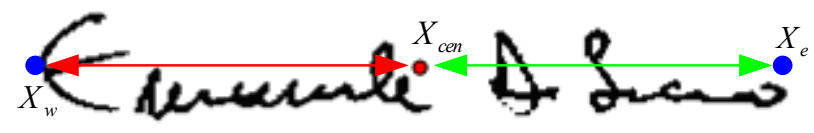

Figure 11: The ratio LR. 
The sixth parameter to extract is represented by the ratio UL:

$$
U L=\frac{Y_{c e n}-Y_{n}}{Y_{s}-Y_{c e n}}
$$

where $Y_{\text {cen }}$ is the vertical coordinate of the centroid, $Y_{n}$ is the upper point of the signature, $\mathrm{Y}_{\mathrm{s}}$ is the lower point of the signature, as shown in fig. 12.

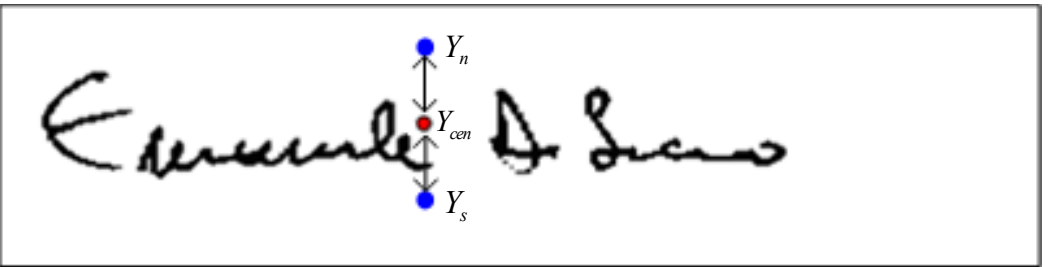

Figure 12: The ratio UL.

The last parameter is represented by the black pixel of the image. The area of each pixel is calculated considering the couple of pixels that surround each pixel, giving a different value according to the following cases:

1) 0 black pixels: area $=0$;

2) 1 black pixel: area $=1 / 4$;

3) 2 adjacent horizontal or vertical pixels: area $=1 / 2$;

4) 2 adjacent diagonal pixels: area $=3 / 4$;

5) 3 black pixels: area $7 / 8$;

6) 4 black pixels: area 1 .

This last parameter is very important since it allows us to consider not only the significant points of a signature but also the pressure used to make the signature. Using this parameter, it is possible to consider an important feature that is generally acquirable by means of digital devices. An example of different pressures is shown in fig. 13.

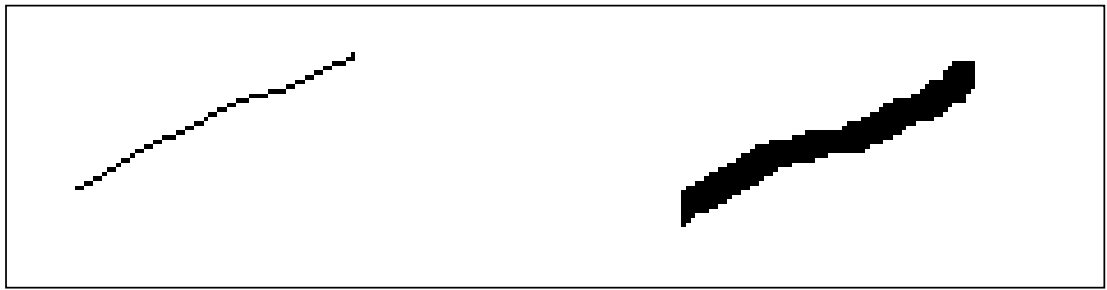

Figure 13: An example of different pressures.

Using these seven parameters it is possible to extract significant aspects of each signatures, as it is shown in the following. In fact, these parameters exalt 
recurrent hidden features of different signatures of the same person. Further, this allows us to represents each signature in a compact way, using a 7 number array more than a $400 \times 100$ pixel image.

\section{The principal components analysis (PCA)}

The principal components analysis (PCA) is a very used technique for the selection and the reduction of the dimension of a set of features. It is based on the correlation principle that allows us to find an orthogonal projection base that allows a reduction of the dimension of features arrays and the reduction of features themselves.

In our case, PCA is used to calculate the projections on the axes in the multidimensional space of the features considering the differences of the signature samples utilized during the training phase.

The used PCA procedure allows a good generalization capability in the reconstruction of a signature when the latter is compared with another signature that has not been used in the training phase.

The used PCA procedure finds some difficulties when it analyses the differences between different signatures belonging to the same person or signatures belonging to different persons, reducing the recognition rate when the signatures classes distribution are separated by co-variance differences more than mean differences.

If $X \in \mathfrak{R}^{N}$ is a numerical array that represents a signature, where $\mathrm{N}$ is the dimension of the space representing the signature features equal to 7 in our case, the PCA procedure finds a proper array P so that:

$$
Y=P^{t} X
$$

obtaining a reduced array $Y \in \mathfrak{R}^{m}$, where $\mathrm{m}<\mathrm{N}$, that captures the most significant features of the original data $\mathrm{X}$.

\section{The whitening transformation}

Once reduced the cardinality of the array related to every signature, the following set of features $Z \in \mathfrak{R}^{m \times n}$ is attained:

$$
Z=\left[Y_{1} Y_{2} \ldots Y_{n}\right]
$$

where $\mathrm{n}$ is equal to the number of signatures used in the training phase.

To optimize the results of the following phases, a proper whitening transformation is applied, obtaining another array $V \in \mathfrak{R}^{m \times n}$ defined as:

$$
V=\Gamma Z
$$

where $\Gamma=\operatorname{diag}\left\{\lambda_{1}^{-1 / 2}, \lambda_{2}^{-1 / 2}, \ldots, \lambda_{m}^{-1 / 2}\right\}$ and $\Gamma \in \mathfrak{R}^{m x m}$ being $\lambda_{1}, \lambda_{2}, \ldots, \lambda_{m}$ the eigenvectors of the array. 


\section{The rotation of the base through Genetic Algorithms}

It is evident that every set of rotation angles corresponds to a different base. To find the optimal base from the classification and generalization point of view between all the rotation transformations, genetic algorithms (GAs) [13] are used.

The evolution of the research of an optimal base starts from an array $\left\{\xi_{1}, \xi_{2}, \ldots, \xi_{m}\right\}$ rotated in a multi-dimensional space with respect to a base $\varepsilon_{1}, \varepsilon_{2}, \ldots, \varepsilon_{m}$ by means of a set of angles $\alpha_{1}, \alpha_{2}, \ldots, \alpha_{m(m-1) / 2}$ where each angles varies between 0 and $\pi / 2$. The goal of GA is to find the optimal set of angles using a proper fitness function that is illustrated in the following.

It is now necessary to define the general chromosome.

10 bit of resolution is used to represents each angle of the base, obtaining a resolution equal to 0.09 degrees per angle: in this way it is necessary to have $[m(m-1) / 2]$ bits to represent all the possible angles.

Since it is necessary to choose between $\mathrm{m}$ arrays of the base, that represent the projections axis, further $\mathrm{m}$ bit have been added to the general chromosome.

The final structure of the chromosome is $\left[\alpha_{1}, \alpha_{2}, \ldots, \alpha_{m(m-1) / 2}, a_{1}, a_{2}\right.$, $\left.a_{m}\right]$. The value of the coefficients $a_{i}, i=1,2, \ldots, m$ is equal to 1 if the chromosome, and the related array, is chosen as an element of the base, 0 otherwise.

The fitness function $\zeta(F)$ drives the evolution of the next generation chromosomes, whose general structure is $F=\alpha_{1}, \alpha_{2}, \ldots, \alpha_{m(m-1) / 2} ; a_{1}, a_{2}, \ldots, a_{m}$, and its structure is:

$$
\zeta(F)=\zeta_{a}(F)+\lambda \zeta_{s}(F)
$$

where $\zeta_{a}(F)$ is the term related to the accuracy of the method while $\zeta_{s}(F)$ is the term related to the separation between the signature classes. $\lambda$ is a positive constant used to balance the influence between the two terms of the fitness function.

In the present work $\zeta_{a}(F)$ is equal to the number of signatures correctly recognized after the rotation and the selection of an axis subset while $\zeta_{s}(F)$ is equal to the measure of the differences between the different classes. $\lambda$ is empirically chosen to let $\zeta_{a}(F)$ be more influent in the next generation selection with respect to $\zeta_{s}(F)$.

In fact, the two terms make opposite direction pressure on the fitness function: $\zeta_{a}(F)$ tends to choose array of the base that guarantee a little difference between the classes while $\zeta_{s}(F)$ makes the opposite. 


\section{Signature recognition}

The recognition is made comparing the array of templates of the signature to be recognized with all the arrays of the other signatures of the database using Euclidian distance: the signature is correctly classified when it reaches the lesser Euclidian distance with respect to a specific signature of the database.

\section{Results}

Our work was made on 600 human signatures, where 500 signatures were used for the training of the genetic algorithm while the remaining 100 signatures were used for the test of the system. The 600 signatures belongs to 100 different persons who gave 6 signatures each ( 2 signs for three different days to consider the unavoidable variation of the sign of the same person)

The proposed system ensures a recognition percentage of $97 \%$ on the considered database. This value can be increased dividing the database into two equal parts, each of them composed by 250 signatures belonging to 50 different persons, and applying the genetic algorithm to this reduced database, using the same signatures for the training phase and for the test phase. In this case a $100 \%$ recognition percentage was reached even in a shorter time, since the algorithm worked on reduced dimension database.

\section{Conclusion}

The proposed human signatures recognition system has resulted to be very versatile and capable of reaching high recognition percentage on large signatures database. It can operate also on more accurate signatures templates based on a greater number of features.

\section{References}

[1] Craner, H. D, and Ostrem, J. S., "Automatic Signature Verification Using a Three-Axis Force-Sensitive Pen”, IEEE Trans. Syst. Man. and Cybernetics, Vol. SMC-13 \#3, 329-337, 1983.

[2] Herbst, N. M., Liu C. N., and Anthony, N. J., "Automatic Signature Verification: System Description and Field Test Results", IEEE Trans. Syst. Man. and Cybernetics, Vol. SMC-9 \#1, 35-48, 1978.

[3] Lorette, G., and Plamondon, R., "Dynamic Approaches to Handwritten Signature Verification", Computer Processing of Handwriting, World Scientific, 21-47, 1990.

[4] Zhao, P., "On Line Signature Verification by Adaptively Weighted DP Matching”, IEICE Trans. Informat. Syst., E79-D(5), 535-541, 1996.

[5] Cho, S. B., "Neural Network Classifier for Recognizing Totally Unconstrained Handwritten Numerals", IEEE Trans. on Neural Networks, vol. 8, 43-53, 1997. 
[6] Leclerc, F., and Plamondon, R., "Automatic Signature Verification: The State of The Art 1989-1993", International Journal of Pattern Recognition and Artificial Intelligence, vol. 8, 643-660, 1994.

[7] Nelson, W., "Statistical Methods for On Line Signature Verification", International Journal of Pattern Recognition and Artificial Intelligence, vol. 8, 749-770, 1994.

[8] Kashi, R. S., "On Line Handwritten Signature Verification Using Stroke Direction Coding”, Optical Engineering, vol. 35, 2526-2533, 1996.

[9] Lee, S., and Pan, J. C., "Off Line Tracing and Representation of Signatures”, IEEE Trans. on Systems, Man, and Cybernetics, vol. 22, 755771, 1992.

[10] Bajaj, R., and Chaudhury, S. "Signature Verification Using Multiple Neural Classifiers", Pattern Recognition, vol. 30, n.1, 1-7, 1997.

[11] Parizeu, M., and Plamondon, R., "A Comparative Analysis of Regional Correlation, Dynamic Time Warping and Skeletal Tree Matching for Signature Verification", IEEE Trans. on Pattern Analysis and Machine Intelligence, vol. 12, n. 7, 1990.

[12] Qi, Y., and Hunt, B., "Signature Verification Using Global and Grid Features", Pattern Recognition, vol. 27, n. 12, 1621-1629, 1994.

[13] Goldberg, D. E., "Genetic Algorithms in Search, Optimization and Machine Learning”, Addison-Wesley, 1989.

[14] Otsu, N., "A threshold selection method from gray-level histograms", IEEE Trans. Sys., Man., Cyber. Vol. 9, 62-66, 1979. 